\title{
Visual Perception During Naturalistic Near-Death Out-of-Body Experiences
}

\author{
Janice Miner Holden, Ed.D. \\ University of North Texas, Denton, TX
}

\begin{abstract}
This study attempted to ascertain the most appropriate content and placement of visual stimuli in a hospital-based study of the veridicality of out-of-body perception in the near-death experience (NDE), and the likelihood that a subject in such a study would notice, clearly perceive, and accurately recall a visual stimulus. Based on the questionnaire responses of 63 subjects who had near-death out-of-body experiences (ND OBEs), at least some of the respondents described sufficiently complete and accurate visual perception during the experience to warrant the pursuit of veridicality research in hospitals. Recommended stimulus content for such research includes a surface with intense color and lighting, and simple number and/or letter combinations. Stimuli should be as far below ceiling level as possible while maintaining double-blind criteria. Some characteristics of the individual NDE and NDEr may affect veridical perception during the ND OBE.
\end{abstract}

The impetus for this research emerged from my previously described rationale for designing a hospital-based study of the veridicality of near-death out-of-body experiences (ND OBEs) (Holden, 1988). The purpose of the study was to ascertain whether ND OB perceptions "correspond precisely to the real (physical) world" (Irwin, 1985, p. 104).

The author gratefully acknowledges the research consultation provided by Northern Illinois University faculty Bruce Kremer, Ed.D.; Robert Karabinus, Ph.D.; Mary Farnum, Ed.D.; Clinton Jesser, Ph.D.; and Thomas Roberts, Ph.D.; access to the IANDS subject pool and research consultation provided by Bruce Greyson, M.D.; and financial support for this research from a Dissertation Completion Award granted by the Graduate School, Northern Illinois University.

Dr. Holden is currently Assistant Professor of Counselor Education at the University of North Texas. This paper was derived in part from her Ed.D. dissertation submitted to the Northern Illinois University Graduate School. Requests for reprints should be addressed to Dr. Holden at the Department of Counselor Education, College of Education, University of North Texas, P.O. Box 13857, Denton, TX 76203. 
The veridicality study was intended to focus on the "naturalistic" ND OBE (Irwin, 1985, p. 7), that stage of the near-death experience (NDE) in which the experiencer finds himself or herself outside of and in the vicinity of the physical body, viewing the physical surroundings, frequently the resuscitation scene (Ring, 1980, pp. 45-53; Sabom, 1982, p. 27).

Following similar out-of-body research designs by Charles Tart (1974) and Karlis Osis $(1974,1975)$, the plan was to place visual stimuli, such as large cards, in the hospital sites in which cardiac arrests most frequently occur: the Emergency Room and the Intensive and Cardiac Care Units. The cards would be produced in such a way that no one would know their exact content, and would be placed in such a way as to be visible only from a vantage point at or near the ceiling, looking down.

With such a double-blind research design, the researcher would have only to wait for patients to be resuscitated from cardiac arrest in any of the target rooms, ask those reporting a naturalistic ND OBE to "guess" the content of the cards in their respective rooms, and then check the accuracy of their answers. If their accuracy rate significantly exceeded the probability of getting the right answer by chance, a strong case could be made that the naturalistic NDE is not imaginal, a case NDErs have been making all along. The use of the term "visual perception" in this research is intended to imply nothing about the nature of the mechanisms that underly the naturalistic ND OBE. The use of the term is based on the reports of naturalistic ND OBErs who describe their subjective experience as one of visual perception.

Problems arose in determining the exact content of the cards, and exactly where the cards should be placed; also in question was whether naturalistic ND OBErs could be expected to notice, clearly perceive, and accurately recall card content of a proposed nature and location. Though NDErs' reference to perceived location and visual perception during the naturalistic ND OBE can be found in the literature of all major researchers of the NDE (Grey, 1985; Moody, 1975; Ring, 1980; Sabom, 1982), no researcher systematically assessed these phenomena. For example, experiencers only sometimes referred directly or indirectly to color perception; a complete profile of color perception was not available. How many recall having seen in color? Among those who did, was all or only part of the color spectrum perceived, and did hues appear the same as or different from those seen while "in body?"

Furthermore, experiencers' spontaneous reports were sometimes contradictory. One experiencer described his naturalistic ND OB vision as identical to vision during normal waking consciousness 
(Sabom, 1982, p. 97), yet others described visual processes that could hardly be considered normal: having seen through solid material (Green \& Friedman, 1983, p. 83), having had $360^{\circ}$ or "panoramic" vision (Moody, 1975, p. 42; Noyes, 1972, p. 176), and having had perfect OB vision despite imperfect vision in the physical body (Ring, 1984, pp. 42-43).

Further indication of the value of a study on naturalistic ND OB visual perception came from the literature on another "altered state of consciousness," dreams (Kahn, Dement, Fisher, \& Barmack, 1962). Whereas a spontaneous dream report contained reference to color $16-23 \%$ of the time, a request that the individual describe a certain object from the dream in more detail yielded reference to color $70-83 \%$ of the time. This is not to equate the naturalistic ND OBE with a dream. But like a person describing a dream, one describing a naturalistic ND OBE may not focus on the process of visual perception during the experience unless asked directly to do so.

The purpose of this study, then, was to describe and analyze several aspects of visual perception during the naturalistic ND OBE. Because a thorough analysis of all aspects of naturalistic ND OB visual perception was beyond the scope of this study, only those aspects that were of particular relevance to the proposed hospital veridicality research were examined.

\section{Method}

\section{Development of the Instrument}

The aspects of naturalistic ND OB vision that the questionnaire was designed to assess were clarity, distortion, color, field of vision, ability to read, completeness and accuracy of memory, attention to extraneous detail, and factors in the enhancement of attention. In order to further analyze these aspects, the questionnaire assessed various characteristics of the individual respondent (gender, current age, age at time of NDE, time elapsed since NDE, recalled OB location, delay of recall of NDE, recalled quality of physical vision at time of NDE, recalled receptivity to interview upon recalling NDE), and of the near-death episode (recalled duration of naturalistic ND OBE, recalled location of physical body during naturalistic ND OBE, recalled amount of light physically present during naturalistic ND OBE, recalled pharmacological influence on physical body during NDE).

A preliminary draft of items was submitted for feedback to a team of 
professional judges, then piloted on three naturalistic ND OBErs who were known to the author. After appropriate revisions, the final instrument consisted of between three and eight Likert-type items for each aspect of visual perception (clarity, distortion, etc.), about half of which were worded in a "positive" direction ("I could see clearly") and the other half "negative" ("My ability to see physical detail clearly was somehow obscured"). These were randomly ordered and followed by 13 items regarding the characteristics of the individual or the near-death episode (gender, age, etc.).

\section{Subjects}

The sample consisted of 100 NDErs who were volunteer research subjects for the International Association for Near-Death Studies (IANDS) and who had answered in the affirmative to a question of whether their NDE had included an out-of-body experience.

\section{Procedures}

A cover letter was composed explaining the purpose of the study. Then 100 questionnaires, cover letters, and postage-paid return envelopes were assembled in unaddressed postage-paid envelopes. These materials were delivered to IANDS at the University of Connecticut, from which the mailing was completed. Subjects were known to the author only as IANDS subject numbers. A follow-up message was mailed three weeks after the date requested for the return of the questionnaires.

\section{Results}

\section{Return Rate}

Of the 100 questionnaires, 77 were returned. Of those, 11 were not completed because the respondents' ND OBEs were of the asensory and/or supernatural type, not the naturalistic type that was the focus of this research. Three additional questionnaires were not usable, yielding 63 usable questionnaires. Of these, four were missing answers to only one or two items from the first section of the questionnaire; these data were filled in by applying the mean. 


\section{Scoring}

Items were reorganized into subtests based on specific aspect of visual perception (clarity, distortion, etc.). Scoring was adjusted so that, regardless of the "positive" or "negative" syntax of the question, responses in a given direction were scored the same. Thus, a response of "strongly agree" to the item "I could see clearly" was scored 5; a response of "strongly disagree" to the item "My ability to see physical detail clearly was somehow obscured" was also scored 5 .

\section{Reliability}

For the purpose of the study, a Cronbach alpha of .80 or higher was considered acceptable, .60-.79 was considered marginal, and .59 or below was considered unacceptable for further statistical analysis. The reliability for each subtest was as follows: clarity (after elimination of one item), alpha = .62; distortion (after elimination of one item), alpha $=.66$; color alpha $=.84$; field of vision (after elimination of one item), alpha $=.65$; reading alpha $=.84$; attention to extraneous detail, alpha $=.91$; attraction of attention, alpha $=.46$.

\section{Descriptive Patterns}

The first task of this study was to formulate descriptive patterns of recalled visual perception. Median, mean, and standard deviation for each aspect of recalled visual perception are reviewed in Table 1.

Of the seven reliable or marginally reliable subtests, five were strongly skewed, with medians above 4.5 , in the direction of recalled clarity of vision, absence of distortion, perception of a complete and accurate color spectrum, complete field of vision, and accuracy of memory. Skewing also occurred, but not so strongly, in the direction of recalled ability to read. Attention to extraneous detail was bimodally distributed, with loading at the extremes of (a) attending exclusively to details of the near-death crisis and of (b) attending equally to extraneous details and to crisis details.

Following are the percentages of responses indicating qualities of naturalistic ND OB vision: was clear (79\%), was free of distortion (76\%), involved acccurate perception of a complete color spectrum (71\%), involved a complete field of vision (77\%), involved a complete and accurate memory of the physical environment (61\%), included the 


\section{Table 1}

Pattern of Recalled Visual Perception

\begin{tabular}{lccc}
\hline & Median & Mean & $\begin{array}{c}\text { Standard } \\
\text { Deviation }\end{array}$ \\
\hline $\begin{array}{l}\text { Aspect } \\
\text { (5 = clarity* }\end{array}$ & 4.715 & 4.245 & 1.209 \\
$\begin{array}{l}\text { distortion* } \\
(5=\text { distortion absent) }\end{array}$ & 4.713 & 4.225 & 1.197 \\
$\begin{array}{l}\text { color } \\
(5=\text { complete \& accurate) }\end{array}$ & 4.508 & 4.014 & 1.238 \\
$\begin{array}{l}\text { field of vision } \\
(5=\text { complete })\end{array}$ & 4.697 & 4.171 & 1.289 \\
$\begin{array}{l}\text { accuracy of memory* } \\
(5=\text { complete \& accurate) }\end{array}$ & 4.531 & 3.937 & 1.319 \\
$\begin{array}{l}\text { ability to read } \\
(5=\text { able to read) }\end{array}$ & 3.875 & 3.688 & .834 \\
$\begin{array}{l}\text { attention to extraneous detail } \\
(5=\text { attentive })\end{array}$ & 3.375 & 3.097 & 1.653 \\
$\begin{array}{l}\text { attraction of attention** } \\
(5=\text { motion/intensity not attractive) }\end{array}$ & 3.950 & 3.633 & 1.399 \\
\hline $\begin{array}{l}\text { Note. } n=63 . \\
* * \text { marginally reliable. } \\
\text { unreliable. }\end{array}$ & & & \\
\hline
\end{tabular}

ability to read (57\%), and involved equal attention both to (a) moving, intensely colored, and/or lighted objects and to (b) stationary and less intensely colored/lighted objects (60\%). More than $48 \%$ of responses indicated equal attention to details of the near-death crisis as to extraneous details during the naturalistic ND OBE, and more than $42 \%$ of responses indicated attention to details of the near-death crisis to the exclusion of extraneous details.

\section{Analyses}

The second task of this study was to determine whether the aspects of naturalistic ND OB visual perception varied based on characteristics of the individual or the near-death episode. 


\section{Main Effects}

In order to maximize identification of possible relationships without undue risk of Type I error, a significance level of .10 was chosen for the reliable subtests. Because of the greater risk of Type 1 error when analyzing the marginally reliable subtests, the more stringent significance level of .05 was chosen.

Follow-up analysis was pursued for all significant results. ANOVAs were subjected to the Cochrans C, Bartlett F, and Max/Min Variance tests to insure compliance with the assumptions of ANOVA; significance at or below the .10 level on at least two of the tests was considered a violation.

Main effects are reviewed in Table 2.

Clarity. No significant difference was found in the recalled clarity of respondents' naturalistic ND OBEs when analyzed by any of the characteristics of the individual or near-death episode.

Distortion. No significant difference was found in recalled distortion of visual perception during the naturalistic ND OBE when analyzed by any of the characteristics of the individual or near-death episode.

Color. Significant differences in recalled completeness and accuracy of the color spectrum during the naturalistic ND OBE were found with only two variables. Further analysis revealed that color perception was apparently more complete and accurate among those respondents who

1. indicated an OB location at or near the ceiling, as compared with an $\mathrm{OB}$ location above the body but not near the ceiling $(p<.05)$, and

2. recalled no pharmacological involvement during the neardeath episodes compared with those who recalled having been under general anesthetic or other pharmacological influence at the time of the NDEs $(p<.10)$.

Field of vision. Significant differences in recalled completeness of field of naturalistic ND OB vision were found with only two variables. Further analysis revealed that the field of vision was apparently perceived as more complete among respondents who

1. indicated an $O B$ location at or near the ceiling, as compared with an $\mathrm{OB}$ location above the body but not near the ceiling $(p<.0001)$, and 


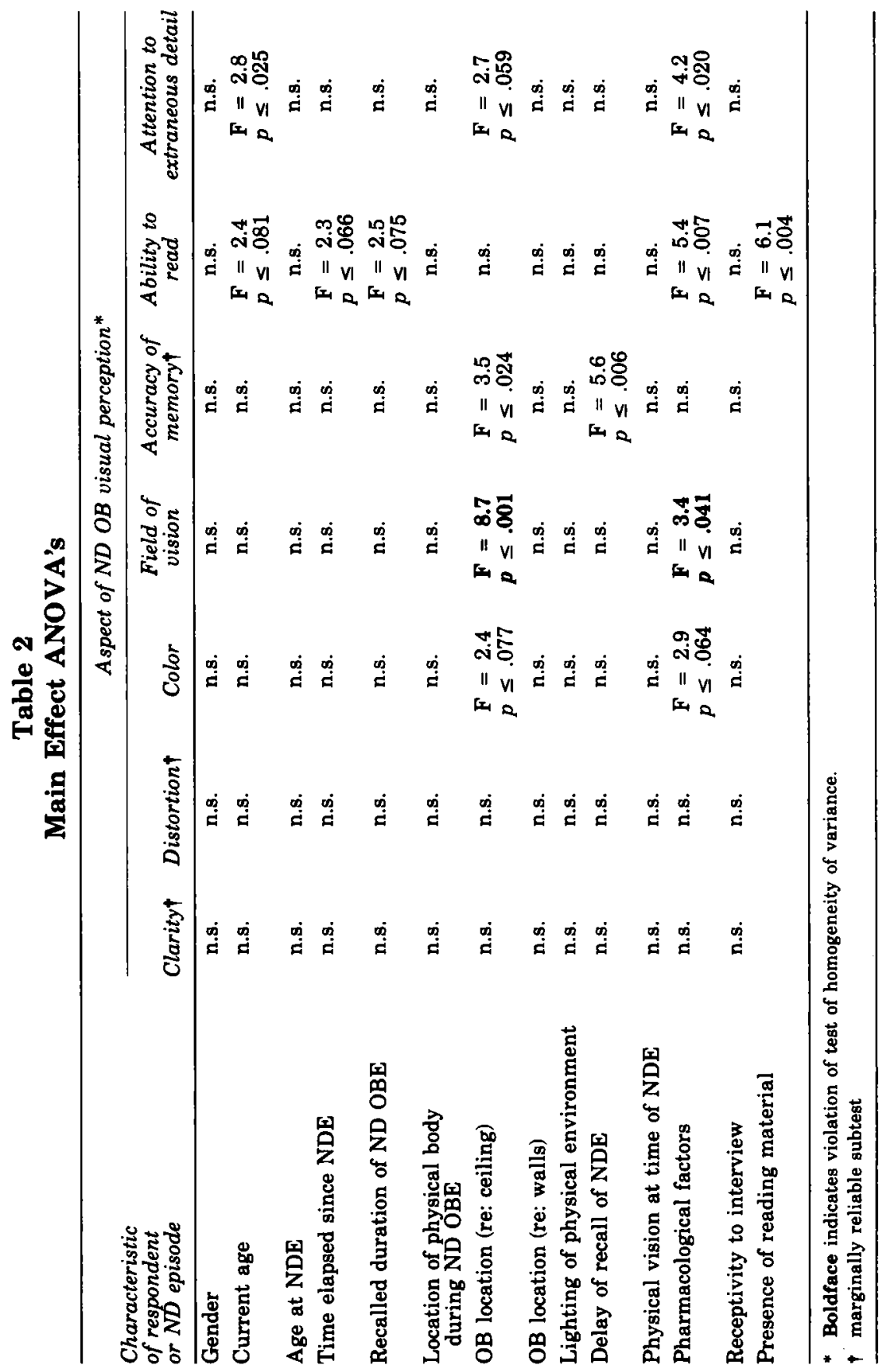


2. recalled no pharmacological involvement during the neardeath episodes, compared with those who recalled having been under general anesthetic or other pharmacological influence at the time of the NDEs $(p<.05)$.

Accuracy of memory. Significant differences regarding recalled completeness and accuracy of memory of the physical environment during the naturalistic ND OBE were found with only two variables. The physical environment was apparently more completely and accurately recalled among those respondents who

1. indicated an $\mathrm{OB}$ location at the ceiling, compared with those indicating an OB location near the ceiling and above the body but not near the ceiling $(p<.01)$, and

2. recalled having remembered the NDE immediately or within a few days of its occurrence, as opposed to recall after some weeks, months, or years had transpired $(p<.01)$.

Attention to extraneous detail. Significant differences in attention to extraneous detail during the naturalistic ND OBE were found with only three variables. Further analysis of $O B$ location relative to the ceiling failed to reveal a significant difference within the groups. Further analysis did, however, reveal an apparently greater attention to extraneous detail among those respondents who

1. were currently under age 60 , when compared with those 60 and older $(p<.0009)$,

2. recalled no pharmacological involvement during their NDEs, compared with those who recalled having been under general anesthetic or some other pharmacological influence $(p<.05)$, and additionally, among those who

3. indicated uncertainty about the duration of their ND OBEs, compared with those who estimated a duration $(p<.015)$.

Ability to Read. Significant differences in the recalled ability to read during the naturalistic ND OBE were found with only five variables. Further analysis revealed that the recalled ability to read was apparently greater among those respondents

1. who were age $34-59$, compared with those younger than 34 and those older than $59(p<.009)$, 
2. whose NDEs occurred 19-29 years ago, compared with those whose experiences occurred in the more recent or more distant past $(p<.004)$

3. who estimated that their naturalistic ND OBEs lasted longer than 10 minutes, compared with those who estimated a duration of less than five minutes $(p<.009)$,

4. who reported the physical presence of reading material at the scene of their near-death episodes, compared with those who reported the absence of, or uncertainty as to the presence of, reading material at the scene $(p<.0008)$, and

5. who recalled no pharmacological involvement during their near-death episode, compared with those who recalled having been under general anesthetic or some other pharmacological influence $(p<.01)$.

The reading scores for those respondents uncertain about the duration of their naturalistic ND OBEs fell about midway between those of respondents estimating a duration of 5 minutes or less and those estimating 10 minutes or more; further analysis indicated no significant difference in recalled ability to read between respondents who estimated a duration and those uncertain about the duration.

\section{Interaction Effects}

Two-way analyses of variance were conducted to determine the interaction of recalled $O B$ location relative to ceiling and recalled $O B$ location relative to wall with all aspects of $N D O B$ visual perception. $A$ significant effect resulted only when analyzed by recalled attention to extraneous detail $(p<.01)$. As a result of further analysis, it appears that those respondents who reported being in the corner and at the ceiling apparently recalled having focused more on crisis details to the exclusion of extraneous details, and those who reported being in the corner and near the ceiling apparently recalled a greater tendency to focus as much on crisis as on extraneous details.

In a further attempt to identify cases in which two independent variables might yield interactive effects with the various aspects of ND OB visual perception, Pearson product-moment coefficients of correlation (two-tailed) were calculated for the independent variables. Some significant and possibly relevant correlations resulted: recalled interview receptivity upon remembering the NDE apparently decreased as time elapsed since NDE increased $(r=.266 ; p=.036)$; recalled inter- 
view receptivity upon remembering the NDE apparently increased as recalled duration of NDE increased $(r=.258 ; p=.042)$; most immediate recall of NDE was apparently associated with body location indoors during entire ND OBE $(r=.318 ; p=.011)$. The latter two relationships seemed worthy of follow-up analyses of variance but yielded nonsignificant results.

\section{Discussion}

Because of some limitations of the questionnaire, three seemingly crucial questions were left unanswered. The first regards $O B$ location relative to the ceiling. In those NDEs that occurred indoors, a perceived locus of consciousness at the ceiling or in an elevated corner of the room was the most frequent location in studies by Kenneth Ring (1980, p. 46) and Margot Grey (1985, p. 34). Michael Sabom's (1982) focus on in-hospital naturalistic ND OBErs specified that "the 'self' which had 'separated' from the unconscious physical body was perceived to be situated above the level of the physical body-a point specifically identified as 'ceiling height' in all but three cases [out of 32]" (pp. 27-28). He clarified that in all three exceptions, the individual was higher than ceiling height.

In this study, therefore, it seemed appropriate to give indoor NDErs the choice of indicating an OB location "at the ceiling," "near the ceiling," "above the body but not near the ceiling," and "other." The "other" distractor was expected to attract a small minority of experients whose OB locations may have seemed parallel to or below the body, or outside of the room. In fact, $21 \%$ of the "indoor NDErs" in this study indicated "other." In every case in which a significant difference was found, the "other" score fell between those of "at the ceiling" and "above the body but not near the ceiling." The meaning of this result, and specifically the makeup of the "other" group, is impossible to determine at this time; future research may elucidate this matter.

A second limitation regards factors in attention. In light of these respondents' apparent tendency to overlook extraneous details in the physical environment, the question of how to enhance attention to a visual stimulus in a hospital veridicality study is of particular interest. Unfortunately, because of the unreliability of the subtest on factors in attention, this research provided no definitive answer to the question of possible enhancement of attention. However, $10 \%$ of responses indicated increased attention to motion, $11 \%$ to intense color, and $24 \%$ to intense lighting. Thus, the current research certainly did not show the 
factors of motion and intensity to be entirely irrelevant to the attraction of visual attention during the naturalistic ND OBE. In this regard, comments offered by three respondents seem appropriate to review. Both a pilot subject and two questionnaire respondents spontaneously commented on the difficulty of answering questions about color because their near-death episodes had taken place in the relatively colorless environment of a hospital room. Thus an intensely colored and lighted stimulus contrasted against the relative "colorlessness" of the typical hospital room may have an attention-enhancing effect. Therefore, a researcher might include these characteristics in the design of a hospital veridicality stimulus.

A third limitation involves the item "I could not see some objects clearly because I was looking through those objects," which was eliminated in order to increase the reliability of the clarity subtest. Disagreement with the item represented, by far, the greatest percentage of responses, but as many as $16 \%$ of responses indicated agreement or strong agreement with this statement. Thus the extent to which veridical perception of a hospital stimulus is threatened by the experiencer "seeing through" it is not known; the possibility of such a threat is disquietingly suggested by these data.

\section{Conclusions}

Because of the self-selected nature of the sample used in this study, it cannot be assumed that the findings of this research are representative of naturalistic ND OB vision in general. However, the following conclusions may be drawn:

1. At least some naturalistic ND OBErs describe sufficiently complete and accurate visual perception during the experience to warrant the pursuit of hospital veridicality research.

2. The recommended stimulus content involves a surface that includes intense color and lighting. Further inclusion of reading content requires many considerations involving analysis of the probability that subjects will accurately report such content (see \#5 below). Nevertheless, as suggested by Tart (personal communication, October 18, 1985), extremely simple number and/or letter combinations should be included for the relatively potent evidential value in those limited cases in which they might be accurately identified. 
3. The placement of the stimulus should be as far below ceiling level as possible while, at the same time, maintaining the criteria for double-blind research.

4. Reported accuracy of recall of such a stimulus so placed may be less likely to differ on the basis of the following characteristics; consequently, the researcher may find it less important to consider these characteristics in deciding upon criteria for potential subjects or in analyzing results: subject's gender, reported $O B$ location relative to the wall(s), or reported quality of physical vision; whether the subject reports his or her physical body to have been in the stimulus-containing room during all or only part of the near-death episode; how brightly or dimly lit the subject describes the physical environment as having been during the naturalistic ND OBE.

5. Reported accuracy of recall of a stimulus might be expected to differ on the basis of the characteristics described below. Therefore, the hospital veridicality researcher is encouraged to determine and adjust expected probability of accurate stimulus identification on the basis of each of these characteristics:

a. Subject's recollection of pharmacological involvement during the near-death episode: Recalled pharmacological involvement may be associated with a lesser tendency for the subject to report that the stimulus fell within his or her field of vision, to report that he or she noticed the stimulus, or to report the correct color and reading content of the stimulus.

b. Subject's reported $O B$ location relative to the ceiling, which may be associated with a lesser tendency for the subject to report that the stimulus fell within his or her field of vision; to report an accurate memory of the stimulus, particularly regarding color content; or possibly to report that he or she noticed the stimulus.

c. Subject's age: Those age 60 or older might be less likely to report having noticed the stimulus than those less than 60 and, along with subjects less than 33 , might be less likely to accurately report the reading content of a stimulus.

d. Subject's report of duration of his or her naturalistic ND OBE: Those reporting a duration of less than 10 minutes might be less likely to report accurately the reading content of the stimulus.

e. How soon after the near-death episode the subject recalled the NDE: Compared to those who remember a naturalistic 
ND OBE within a few days of a near-death episode, subjects who do not might be less likely to report a complete and accurate memory of the physical environment in which the near-death episode occurred, including their memory of the stimulus.

6. Considering that naturalistic ND OB visual perception may be better in this sample than among the entire population of experiencers; that, even among this sample, reported willingness to be interviewed decreased as recalled duration of the experience decreased; and that many probable and unavoidable limitations to accurate naturalistic ND OB perception of a visual stimulus were found even among those in this sample who were reportedly willing to be interviewed once they remembered their experiences, the process of accumulating sufficient data in hospital veridicality research may be protracted. Thus it is recommended that such research be conducted simultaneously at more than one hospital.

\section{References}

Green, J. T., \& Friedman, P. (1983). Near-death experiences in a Southern California population. Anabiosis: The Journal of Near-Death Studies, 3, 77-95.

Grey, M. (1985). Return from death: An exploration of the near-death experience. London, England: Routledge \& Kegan Paul.

Holden, J. M. (1988). Rationale and considerations for proposed near-death research in the hospital setting. Journal of Near-Death Studies, 7, 19-31.

Irwin, H. J. (1985). Flight of mind: A psychological study of the out-of-body experience. Metuchen, NJ: Scarecrow Press.

Kahn, E., Dement, W., Fisher, C., \& Barmack, J. E. (1962). Incidence of color in immediately recalled dreams. Science, $32,1054-1055$.

Moody, R. A., Jr. (1975). Life after life. Covington, GA: Mockingbird.

Noyes, R. (1972). The experience of dying. Psychiatry, 35, 174-184.

Osis, K. (1974). Perspectives for out-of-body research. In W. G. Roll, R. L. Morris, \& J. D. Morris (Eds)., Research in parapsychology, 1973 (pp. 110-113). Metuchen, NJ: Scarecrow Press.

Osis, K. (1975). Perceptual experiments on out-of-body experiences. In J. D. Morris, W. G. Roll, \& R. L. Morris (Eds.), Research in parapsychology, 1974 (pp. 53-55). Metuchen, NJ: Scarecrow Press.

Ring, K. (1980). Life at death: A scientific investigation of the near-death experience. New York, NY: Coward, McCann \& Geoghegan.

Ring, K. (1984). Heading toward omega: In search of the meaning of the near-death experience. New York, NY: William Morrow.

Sabom, M. B. (1982). Recollections of death: A medical investigation. New York, NY: Harper \& Row.

Tart, C. T. (1974). Out-of-the-body experiences. In E. D. Mitchell (Ed.), Psychic exploration: A challenge for science. New York, NY: G. P. Putnam \& Sons. 